phù hợp với kết quả về những câu hỏi mà các bác sỹ thường gặp ở các bệnh nhân GERD. Ngoài ra, các bác sỹ cũng kỳ vọng đây sẽ là một kênh tương tác giữa bác sỹ và bệnh nhân từ đó đảm bảo bệnh nhân được tối ưu hóa trong tư vấn và theo dõi điều trị.

Đây là khảo sát bước đầu với số lượng bác sỹ tham gia còn hạn chế. Tuy nhiên, khảo sát cũng đã cung cấp cái nhìn ban đầu về thực trạng và nhu cầu sử dụng ƯDDĐnhằm hỗ trợ quản lý dạ dày-thực quản, là tiền đề cho các nghiên cứu tiếp theo nhằm làm rõ hơn nhu cầu thực tế trong sử dụng ứng dụng.

\section{KẾT LUÂ̂N}

Tỷ lệ các bác sỹ từng điều trị cho bệnh nhân GERD chiếm đến $98 \%$ tổng số bác sỹ tham gia khảo sát, $88 \%$ bác sỹ đã từng gặp bệnh nhân GERD kháng trị. $100 \%$ bác sỹ đồng ý xây dựng một U'DDĐđể hỗ trợ quản lý GERD. Bên cạnh đó, ứng dụng hỗ trợ quản lý trào ngược dạ dàythực quản sẽ là một hướng đi đầy hứa hẹn giúp nâng cao chất lượng điều trị cho bệnh nhân.

\section{TÀI LIẸU THAM KHẢO}

1. Tổ chức $\mathbf{Y}$ tế thế giới, Tổng quan quốc gia về Nhân lực y, tế Việt Nam 2018.

2. Bộ Y Tuế. Ứng dụng công nghệ thông tin hướng tới xây dựng nền y tế thông minh. 2019; Available from: https://moh.gov.vn/chuong-trinh-muc-tieuquoc-gia/ /asset_publisher/ 7ng11fEWgASC/ content/ung-dung-cong-nghe-thong-tin-huong-toixay-dung-nen-y-te-thong-minh.

3. Đặng Thị Lõn, et al., Hình thái vùng nối dạ dày thự̛c quản và ap lực cơ thắt thực quản dưới bằng kỹ thuât HRM ở bệnh nhân có thooát vị hoành trượt trên nội soi. Tạp chí Y Dược học Lẩm sàng 108 2020. 15(2).

4. Đào Việt Hằng and Hoàng Bảo Long, Bước đầu đánh giá kết quả đo $\mathrm{pH}$-trở kháng 24 giờ ở bênh nhân trào ngược da dày-thức quản kháng tri. Tạp chí nghiên cứu Ý học, 2019. 119(3): p. 33-40.

5. Vakil, N., et al., The Montreal definition and classification of gastroesophageal reflux disease: a global evidence-based consensus. Am J Gastroenterol, 2006. 101(8): p. 1900-20; quiz 1943.

6. Fock, K.M., et al., Asia-Pacific consensus on the management of gastro-oesophageal reflux disease: an update focusing on refractory reflux disease and Barrett's oesophagus. Gut, 2016. 65(9): p. 1402-15.

7. Mermelstein, J., A. Chait Mermelstein, and M.M. Chait, Proton pump inhibitor-refractory gastroesophageal reflux disease: challenges and solutions. Clin Exp Gastroenterol, 2018. 11: p. 119-134.

8. El-Serag, H., A. Becher, and R. Jones, Systematic review: persistent reflux symptoms on proton pump inhibitor therapy in primary care and community studies. Aliment Pharmacol Ther, 2010. 32(6): p. 720-37.

9. Mermelstein, J., A.C. Mermelstein, and M.M. Chait, Proton pump inhibitors for the treatment of patients with erosive esophagitis and gastroesophageal reflux disease: current evidence and safety of dexlansoprazole. Clin Exp Gastroenterol, 2016. 9: p. 163-72.

\title{
ĐÁNH GIÁ KẾT QUẢ ĐO NHÃN ÁP BẰNG VỚI MộT SỐ LOẠI NHÃN ÁP KẾ
}

\section{Đỗ Tấn ${ }^{1}$, Phạm Thị Thu Thủy ${ }^{2}$, Hoàng Thị lành ${ }^{3}$}

\section{TÓM TẮT}

Mục tiêu: Đánh kết quả đo nhãn áp (NA) bằng nhãn áp kế (NAK) Maclakov, NAK Goldmann và NAK không tiếp xúc. Đối tượng và phương pháp nghiên cứu: Nghiên cứu mô tả cắt ngang trên 702 mắt của 352 bệnh nhân khám và điều trị tại khoa Glôcôm Bệnh viện Mắt Trung ương từ tháng 11/2017 đến tháng 8/2018. Kết quả: Tuổi trung bình của nhóm bệnh nhân trong nghiên cứu là 56,06 $\pm 16,88$ (khoảng, 15 đến 90 ). Nhóm tuổi từ 60 - $\leq 80$ có tý lệ

${ }^{1}$ Bệnh Viện Mắt Trung Ương

Đại Học Y Hà Nọi

${ }^{3}$ Bềnh Viện Thanh Nhàn

Chịu trách nhiệm chính: Đỗ Tấn

Email: dotan20042005@yahoo.com

Ngày nhận bài: 18.12.2020

Ngày phản biện khoa họ: 25.01.2021

Ngày duyệt bài: 2.2.2021 cao nhất chiếm 42,3\%. Bênh nhân nữ chiếm tỷ lệ cao hơn (55,4\%). Trong số bệnh nhân nghiên cứu, glôcôm chiếm tỷ lệ cao nhất 42,5\%. Với kết quả đo của cả ba loại NAK, các mắt nhóm glôcôm có NA trung bình cao nhất, các mắt bong võng mac có NA trung bình thấp nhất. Với NAK Maclakov, nhóm có NA trong khoảng từ 16 đến $21 \mathrm{mmHg}$ chiếm tỷ lề cao nhất $(74,1 \%)$, các mắt có $\mathrm{NA}$ rất cao $\geq 32 \mathrm{mmHg}$ có tỷ lệ thấp nhất 2,6\%. Chênh lệch chỉ số $N A$ của NAK Maclakov và NAK Goldmann với từng mức NA là: NA thấp < $16 \mathrm{mmHg}: 2,397 \pm 1,248 \mathrm{mmHg}$; Bình thường 16 - 21mmHg: 2,604 $\pm 1,563 \mathrm{mmHg}$; Bình thường cao 22 - 25mmHg: 1,100 $\pm 3,460 \mathrm{mmHg}$; Cao $26-31$ mmHg: $-2,043 \pm 3,989 \mathrm{mmHg}$; Rất cao $\geq 32 \mathrm{mmHg}$ : $3,611 \pm 4,667 \mathrm{mmHg}$. Chênh lệch chỉ số NA của NAK Maclakov và NAK không tiếp xúc với từng mức NA là: $\mathrm{NA}$ thấp < 16mmHg: $3,687 \pm 2,741 \mathrm{mmHg}$; Bình thường 16-21mmHg: 2,270 $\pm 3,070 \mathrm{mmHg}$; Bình thường cao $22-25 \mathrm{mmHg}:-0,536 \pm 4,960 \mathrm{mmHg}$; Cao $26-31 \mathrm{mmHg}:-3,207 \pm 5,932 \mathrm{mmHg}$; Rất cao $\geq$ 32 mmHg: $-3,883 \pm 4,913$ mmHg. Kết luận: Kết quả 
đo NA ở các nhóm bệnh khác nhau thường khác nhau. Giá trị đo đước của các loai NAK khác nhau có thể chệnh lệch khá nhiều, mức độ chênh lệch, chiều chểnh lệch thay đổi theo miền NA.

Tư khóa: Nhán áp (NA), Nhãn áp kễ (NAK) Maclakov, NAK Goldmann, NAK không tiếp xúc.

\section{SUMMARY \\ EVALUATING THE IOP READINGS FROM DIFFERENT TONOMETERS}

Purpose: To analyze IOP readings with Maclakov tonometer, Goldmann applanation tonometer (GAT) and non-contact airpuff tonometer. Subjects and methods: A cross sectional study over 702 eyes of 352 patients treated at Glaucoma Deparment, Vietnam National institute of Ophthamology (VNIO) from November 2017 to August 2018. Results: The mean age of studied group was $56,06 \pm 16,88$ year old (range, 15 to 90). Subgroup of 60 to 80 year old was most popular accounting for $42,3 \%$. Female patients were $55,4 \%$. Glaucoma patients were at highest rate $(42,5 \%)$. With all 3 tonometers, Glaucoma group had highest mean IOP while retinal detachment group had lowest. With Maclakov tonometer group with IOP from 16 to $21 \mathrm{mmHg}$ accounted most $(74,1 \%)$, while very high (IOP $\geq 32 \mathrm{mmHg}$ ) group accounted least $(2,6 \%)$. The IOP reading difference between Maclakov and Goldmann tonometers depended on IOP intervals: Lower IOP < $16 \mathrm{mmHg}$ : 2,397 $\pm 1,248 \mathrm{mmHg}$; Normal IOP (16 - $21 \mathrm{mmHg}): 2,604 \pm 1,563 \mathrm{mmHg}$; higher IOP $(22-25 \mathrm{mmHg}): 1,100 \pm 3,460 \mathrm{mmHg}$; high IOP $(26-$ $31 \mathrm{mmHg}):-2,043 \pm 3,989 \mathrm{mmHg}$; Very high IOP $\geq 32$ mmHg: $-3,611 \pm 4,667 \mathrm{mmHg}$. Similary, the IOP reading difference between Maclakov and noncontact airpuff tonometers depended on IOP intervals: Lower IOP < $16 \mathrm{mmHg}: 3,687 \pm 2,741 \mathrm{mmHg}$; normal IOP: 16 - $21 \mathrm{mmHg}: 2,270 \pm 3,070 \mathrm{mmHg}$; higher IOP: 22 25mmHg: $-0,536 \pm 4,960 \mathrm{mmHg}$; high IOP: $26-31$ $\mathrm{mmHg}:-3,207 \pm 5,932 \mathrm{mmHg}$; very high IOP $\geq 32$ mmHg: $-3,883 \pm 4,913 \mathrm{mmHg}$. Conclusions: IOP results were different from different disease groups. IOP readings from different tonometers could be different and the magnitude, way of difference may depend on the IOP intervals.

Keywords: intraocular, Maclakov tonometer, Goldmann applanation tonometer, non-contact airpuff tonometer.

\section{I. ĐẶT VẤN ĐỀ}

NA là một yếu tố sinh lý rất quan trọng trong việc duy trì cấu trúc và chức năng của nhã̃n cầu. Việc xác định chính xác trị số NA là rất quan trọng, luôn được các bác sĩ nhãn khoa quan tâm.

NAK là dụng cụ đo áp lực nội nhãn một cách gián tiếp. Tùy theo sự thay đổi hình dạng giác mạc khi đo, người ta chia ra 2 loại NAK: loại ấn Iõm và loại đè phẳng. NAK đè phẳng gồm hai loại: loại có lực ép cố định như NAK Maclakov và loại có diện tích vùng đè phẳng cỗ định như NAK Goldmann và NAK không tiếp xúc [1]. NAK Maclakov có ưu điểm là gọn, dể sử dụng, rẻ tiền và kết quả có thể lưu lại. NAK Goldmann kết quả đo được thường chính xác và được xem như tiêu chuẩn vàng trong đo NA. NAK không tiếp xúc được sử dung rộng rãi vì dễ sử dụng, không làm lây lan nhiếm trùng như các NAK tiếp xúc, không cần dùng thuốc tê, không gây trầy xước giác mạc [1]. Không có một loại NAK nào có thể sử dụng cho tất cả các bệnh nhân, trong mọi hoàn cảnh, vì vậy, tùy từng trường hợp mà sử dụng loại NAK phù hợp. Trong khi đó kết quả đo giữa các NAK không đồng nhất, NAK Maclakov đã được nghiên cứu khá nhiều, việc tìm mối tương quan giữa chỉ số NA của 3 loại NAK là rất cần thiết trong thực hành lâm sàng. Vì vậy chúng tôi tiến hành thực hiện đề tài với mục tiêu: So sánh kêt quả thu được giữa NAK Maclakov với NAK Goldmann và NAK Không tiếp xúc.

\section{II. ĐỐI TƯợNG VÀ PHƯƠNG PHÁP NGHIÊN CỨU \\ Đối tượng nghiên cứu: \\ Tiêu chuẩn lựa chơn: Các bênh nhân từ 15} tuổi trở lên, không có chống chỉ định đo $N A$, đồng ý tham gia nghiên cứu.

Tiêu chuẩn loại trừ: Bênh nhân đang có tình trạng viêm ở mắt; các bệnh lý ở giác mạc như sẹo, loạn dưỡng, tróc biểu mô, tân mạch, giác mạc chóp. Bệnh nhân mới trải qua phẫu thuật nội nhãn < 7 ngày, tiền sử phẫu thuật trên giác mạc (gồm cả phẫu thuật lasik); bệnh nhân co thắt mi, rung giật nhãn cầu; dị ứng thuốc tê nhỏ mắt.

\section{Phương pháp nghiên cứu:}

- Thiết kế nghiên cứu: Nghiên cứu mô tả cắt ngang.

- Cõ̃ mẫu được tính theo công thức

$$
n=Z^{2} 1-a / 2 \frac{s^{2}}{\Delta^{2}}
$$

n Cõ mấu nghiên cứu cần có

s: độ lệch chuẩn (ước tính từ nghiên cứu trước) chọn $\mathrm{s}=2,66$ [2]

$\Delta$ : sai số ước lượng $=0,2$

a: mức ý nghĩa thống kê

$Z_{1-a / 2}=1,96$ với $a=0,05$

Vậy cỡ mẫu tối thiểu là $n=680$ mắt. Chọn $n$ $=702$ mắt

- Quy trình nghiên cứu

- Lựa chọn bệnh nhân, hỏi bệnh, khám bệnh, làm bệnh án nghiên cứu.

- Đo NA với NAK không tiếp xúc, mỗi mắt đo 3 lần, máy tự tính ra kết quả trung bình cộng.

- Cho bệnh nhân nghỉ 10 phút trước khi đo bằng NAK Goldmann được đo bởi chính người thức hiên đề tài, không biết kết quả đo NA hơi. Mỗi mắt được đo hai lần liền nhau, nếu kết quả trong hai lần chênh lệch không quá $2 \mathrm{mmHg}$ sẽ 
được chấp nhận với kết quả lấy trung bình cộng của hai lần đó.

- Cho bệnh nhân nghỉ 10 phút trước khi đo bằng NAK Maclakov thực hiện bởi 1 điều dưỡng kinh nghiệm, không biết kết quả đo 2 NAK trước đó: dùng quả cân $10 \mathrm{~g}$ và thuốc nhuộm bằng Prolacgol- glycerin được pha theo tiêu chuẩn. Chỉ lây những kết quả khi diện tiếp xúc in ra giấy có hình tròn đều, cân xứng và nằm chính giữa mặt quả cân.

- Ghi nhận kết quả NA của mỗi loại NAK

- Xử lý số liệu: Theo phần mềm thống kê y học SPSS 16.0.

\section{KẾT QUẢ NGHIÊN CỨU}

Đặc điểm chung của nhóm nghiên cứu. Nghiển cứu được thực hiện trên 702 mắt của 352 bệnh nhân với giới tính nữ chiếm tỷ lệ cao hơn $(55,4 \%)$, nam chiếm 44,6\%. Nhóm bệnh nhân nghiên cứu tập trung chủ yếu trong nhóm trên 40 tuổi (chiếm 82,7\%). Tuổi thấp nhất trong nhóm nghiên cứu là 15 tuổi, cao nhất là 90 tuổi, tuổi trung bình là $56,06 \pm 16,88$. Các mắt glôcôm chiếm tỷ lệ $42,3 \%, 24,1 \%$ mắt được chẩn đoán đục thủy tinh thể; các mắt đã đặt thủy tinh thể nhân tạo, có các bệnh lý đáy mắt (lỗ hoàng điểm, màng trước võ̃ng mạc) xuất huyết dịch kính được xếp vào nhóm bệnh khác chiếm tỷ lệ 13,4\%; 9,3\% mắt bị bong võng mạc và $6,8 \%$ mắt bình thường.

Kết quả đo NA với 3 loại NAK. Với kết quả đo của cả ba loại NAK, các mắt nhóm glôcôm có NA trung bình cao nhất, các mắt bong võng mạc có NA trung bình thấp nhất. Kiểm định ANOVA, thấy sự khác biệt về trung bình NA của các nhóm Glôcôm với nhóm các bệnh khác có ý nghĩa thống kê với $p=0,0001$.

Bảng 1. NA trung bình của từng loại NAK theo bệnh

\begin{tabular}{|c|c|c|c|}
\hline Chẩn đoán & $\begin{array}{c}\text { NAK Maclakov } \\
\text { (mmHg) }\end{array}$ & $\begin{array}{c}\text { NAK Goldmann } \\
(\mathbf{m m H g})\end{array}$ & $\begin{array}{c}\text { NAK không tiếp } \\
\text { xúc( } \mathbf{m m H g} \text { ) }\end{array}$ \\
\hline Bình thường & $16,98 \pm 1,50$ & $14,61 \pm 1,81$ & $14,79 \pm 3,52$ \\
\hline Đục thưy tinh thế & $17,06 \pm 2,14$ & $14,50 \pm 2,53$ & $14,03 \pm 3,71$ \\
\hline Glôcôm góc đóng NP & $19,87 \pm 5,61$ & $18,31 \pm 8,13$ & $17,32 \pm 6,31$ \\
\hline Glôcôm góc mở NP & $21,38 \pm 5,11$ & $20,32 \pm 7,20$ & $21,59 \pm 8,79$ \\
\hline Glôcôm thứ phát & $21,83 \pm 6,76$ & $21,41 \pm 9.52$ & $20,71 \pm 9,14$ \\
\hline Bong võng mạc & $16,28 \pm 1,93$ & $13,86 \pm 2,56$ & $14,25 \pm 4,10$ \\
\hline Bệnh khác & $17,83 \pm 2,99$ & $14.96 \pm 3.75$ & $15,04 \pm 4,12$ \\
\hline
\end{tabular}

Với mức NA $<16 \mathrm{mmHg}$, từ $16-21 \mathrm{mmHg}$ Những mắt có trong nhóm NA từ $22 \mathrm{mmHg}$ trở và từ 22 đến $25 \mathrm{mmHg}$ khi đo bằng NAK lên NA trung bình khi đo bằng NAK không tiếp Maclakov, NA trung bình của NAK Maclakov cao xúc cao hơn khi đo bằng NAK Maclakov. Như vậy hơn NAK Goldmann. Với mức NA $\geq 26 \mathrm{mmHg}$ NAK không tiếp xúc có giá trị NA trung bình cao NA trung bình của NAK Goldmann cao hơn NAK Maclakov. Các nhóm có NA khi đo bằng NAK Maclakov $<22 \mathrm{mmHg}$ có giá trị NA trung bình của NAK Maclakov cao hơn NAK không tiếp xúc. hơn NAK Goldmann với những mắt có NA > 21 $\mathrm{mmHg}$ khi đo bằng NAK Maclakov. NA trung bình khi đo bằng NAK Goldmann và NAK không tiếp xúc chênh lệch nhau ít hơn so với NAK Maclakov.

Bảng 2. NA trung binh của 3 loai NAK theo từng múc NA

\begin{tabular}{|c|c|c|c|}
\hline $\begin{array}{c}\text { NA trung bình NAK } \\
\text { Maclakov }(\mathbf{m m H g})\end{array}$ & $\begin{array}{c}\text { NA trung bình NAK } \\
\text { Maclakov }(\mathbf{m m H g})\end{array}$ & $\begin{array}{c}\text { NA trung bình NAK } \\
\text { Goldmann } \mathbf{( m m H g})\end{array}$ & $\begin{array}{c}\text { NA trung bình NAK } \\
\text { không tiếp xúc } \mathbf{( m m H g})\end{array}$ \\
\hline $\mathbf{2 1 6}$ & $14,18 \pm 1,21$ & $11,78 \pm 1,98$ & $10,49 \pm 3,20$ \\
\hline $\mathbf{1 6 - 2 1}$ & $17,27 \pm 1,41$ & $14,67 \pm 1,96$ & $15,00 \pm 3,49$ \\
\hline $\mathbf{2 2 - 2 5}$ & $23,64 \pm 1,31$ & $22,54 \pm 4,16$ & $24,18 \pm 5,54$ \\
\hline $\mathbf{2 6 - 3 1}$ & $28,04 \pm 1,58$ & $30,09 \pm 4,80$ & $31,35 \pm 6,38$ \\
\hline$\geq \mathbf{3 2}$ & $35,17 \pm 2,46$ & $38,78 \pm 5,23$ & $39,05 \pm 5,59$ \\
\hline
\end{tabular}

Khi tách riêng từng mức NA, sự chênh lệch của NA trung bình khi đo bằng NAK Maclakov và NAK Goldmann ở mỗi mức khác nhau, sự chênh lệch ít nhất là $1,100 \pm 3,460 \mathrm{mmHg}$ với nhóm NA từ $22-25 \mathrm{mmHg}$, chênh lệch nhiều nhất là $3,611 \pm 4,667 \mathrm{mmHg}$ với nhóm $\mathrm{NA} \geq 32 \mathrm{mmHg}$. Tương tự, sự chênh lệch của NA trung bình khi đo bằng NAK Maclakov và NAK không tiếp xúc ở mỗi mức khác nhau, sự chênh lệch ít nhất là $0.536 \pm 4,960 \mathrm{mmHg}$ với nhóm NA từ $22-25$ $\mathrm{mmHg}$, chênh lệch nhiều nhất là $-3,883 \pm 4,913$ $\mathrm{mmHg}$ với nhóm $\mathrm{NA} \geq 32 \mathrm{mmHg}$. Ở mức $\mathrm{NA}$ khoảng $25 \mathrm{mmHg}$ thì kết quả đo của NAK Maclakov và NAK Goldmann tương đương nhau. Dưới mức này kết quả đo NAK Maclakov cao hơn; ngược lại, trên mức này kết quả đo NAK 
Maclakov thấp hơn NAK Goldmann. Ở mức NA khoảng $22 \mathrm{mmHg}$ thì kết quả đo của NAK Maclakov và NAK không tiếp xúc tương đương nhau. Dưới mức này kết quả đo NAK Maclakov cao hơn; ngược lại, trên mức này kết quả đo bằng NAK Maclakov thấp hơn NAK không tiếp xúc. NAK Goldmann và NAK không tiếp xúc cho kết quả khá tương đồng ở các mức NA.

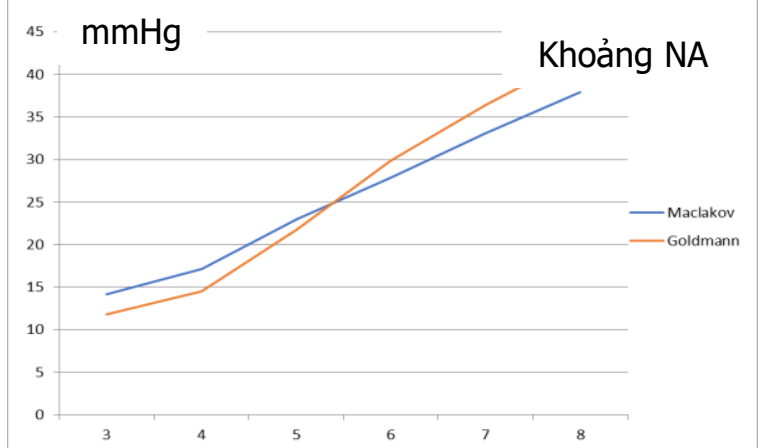

Biểu đồ 1: NA trung bình với NAK Maclakov và NAK Goldmann theo từng khoảng NA

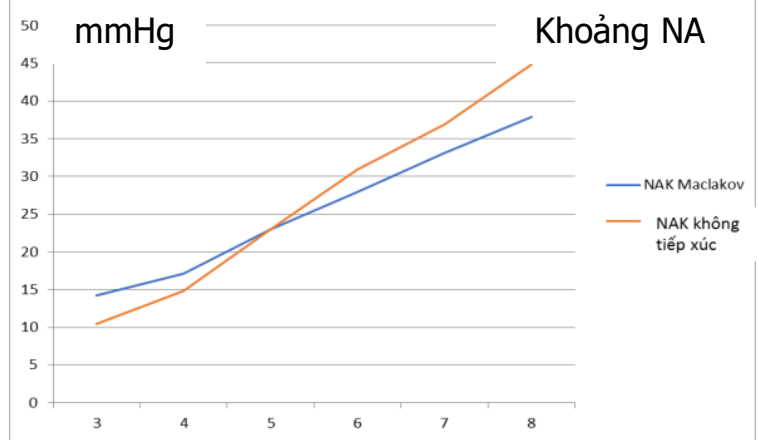

Biểu đồ 2: NA trung bình với NAK Maclakov và NAK không tiếp xúc theo từng khoảng NA (khoảng $5 \mathrm{mmHg}$ )

\section{BÀN LUẬN}

Theo tác giả Nguyễn Thị Thanh Thu, NA trung bình khi đo bằng NAK Goldmann nhỏ hơn khi đo bằng NAK Maclakov quả cân 10 gam là $3,09 \pm 1,70 \mathrm{mmHg}$ [2]. Trong một nghiên cứu của Ayres vào năm 1968, tác giả đã tiến hành đo NA cho 50 người trưởng thành (100 mắt) bằng NAK Goldmann và NAK Maclakov. Kết quả thu được: giá trị NA trung bình thu được khi đo bằng NAK Goldmann nhỏ hơn so với khi đo bằng NAK Maclakov các quả cân 5 gam; 7,5 gam; 10 gam lần lượt là 0,$29 ; 1,28$ và $2,7 \mathrm{mmHg}$ [3]. Nghiên cứu của Võ Đức Dũng 2005 cũng cho kết quả tương tự [4]. Như vậy, kết quả sự chệnh lệch NA trung bình khi đo bằng NAK Goldmann và NAK Maclakov quả cân $10 \mathrm{gam}$ của chúng tôi thấp hơn các tác giả trên. Có thể nguyên nhân là do trong đối tượng nghiên cứu của chúng tôi có cả những mắt NA cao, khi đó NAK Goldmann cho kết quả đo NA cao hơn NAK Maclakov, vì vậy hiệu số chênh lệch mang giá trị âm làm giảm chênh lệch NA của toàn bộ nhóm nghiên cứu.

Với các nhóm có giá trị NA đo bằng NAK Maclakov ở mức cao > $25 \mathrm{mmHg}$, khi NA càng cao sự chênh lệch của NAK Maclakov và NAK Goldmann càng nhiều. Cụ thể với các mắt có mức NA từ 26 đến $31 \mathrm{mmHg}$ sự chênh lệch kết quả đo của NAK Maclakov với NAK Goldmann là $2,043 \pm 3,989$, trong khi đó với mức NA từ 32 $\mathrm{mmHg}$ trở lên sự chênh lệch này lên đến $-3,611$ $\pm 4,667 \mathrm{mmHg}$. Và rõ ràng là sự khác biệt giữa 2 loại NAK này là sự khác biêtt 2 chiều phụ thuộc và miền NA. Tại miền NA thấp hơn thì triwj số đo được của NAK Goldmann thường thấp hơn NAK Maclakov, trong khi đó tại miền NA cao 25 $\mathrm{mmHg}$, NAK Goldmann lại cho ra trị số đo được cao hơn NAK Maclakov. Để lý giải điêuu này chúng tôi cho rằng sự khác biệt nằm ở nguyên lý đo của từng loại NAK: NAK Malakov dựa trên lực đo (khối lượng) cố định sau đó ước lượng trị số NA dựa vào diên tích đè det. Còn NAK Goldmann lại có diện tích dè dẹt cố định (đường kính 3,06 $\mathrm{mm}$ ) và ước lượng NA dựa và lực đè dẹt. Như vậy tại miền NA thấp khi nhãn cầu bị đè dẹt nhiều, gây tăng NA giả tạo và giá trị đo được từ NAK Maclakov sẽ cao hơn thực tế.

Trong nghiên cứu của chúng tôi, chênh lệch NA trung bình giữa NAK Maclakov và NAK khổng tiếp xúc của toàn bộ nhóm nghiên cứu là 1,693 $\pm 3,952 \mathrm{mmHg}$. Kết quả mức chênh lệch của chúng tôi thấp hơn so với tác giả Phạm Minh Đức, theo tác giả này NA đo bằng NAK Maclakov cao hơn NA đo bằng NAK không tiếp xúc trung bình là $2,87 \pm 0,89 \mathrm{mmHg}$ [5]. Nếu chỉ xét nhóm các mắt có NA nằm trong giới hạn bình thường 16 - $21 \mathrm{mmHg}$ kết quả của chúng tôi tương tự với tác giả Phạm Minh Đức, sự chênh lệch của NAK Maclakov với NAK không tiếp xúc là 2,270 \pm $3,070 \mathrm{mmHg}$, nguyên nhân do chúng tôi lấy cả những mắt NA cao, hiệu số chênh lệch mang giá trị âm nên đã làm hạ giá trị chênh lệch giữa hai NAK của toàn bộ nhóm nghiên cứu. Tương tự như với sự khác biệt với NAK Goldmann, sự khác biệt về giá trị đo của NAK Maclakov với NAK không tiếp xúc cũng phụ thuộc miền NA. Tại miền NA thấp hơn $22 \mathrm{mmHg}$, NA đo được với NAK không tiếp xúc thường thấp hơn NAK Maclakov, ngược lại, tại miền NA cao hơn $22 \mathrm{mmHg}$, NAK không tiếp xúc lại cho ra trị số đo cao hơn. Lý giải về điều này, chúng tôi cũng cho rằng sự khác biệt về giá trị đo được giữa 2 loại 
NAK cũng phu thuộc vào sư khác nhau về nguyên lý đo. NAK không tiếp xúc (phụt hơi) dựa trên sự đè dẹt của giác mạc trung tâm do luồng hơi phát ra từ đầu đo, diển tích đè dẹt cố định có đường kính 3,06 mm rất giống với nguyên lý đo của NAK Goldmann. Cũng chính vì lý do này giá trị đo của NAK Goldmann và NAK không tiếp xúc khá tương đồng nhau ở các miền NA.

\section{KẾT LUÂ̂N}

Trị số NA đo được với NAK Maclakov tương đối khác biệt với NAK Goldmann, NAK không tiếp xúc và sự khác biệt này phụ thuộc miền NA. Trong thực hành lâm sàng cần lưu ý đến sự khác biệt này và không nên ngoại suy giá trị của NAK này cho NAK kia theo ngoại suy tuyến tính.
TÀI LIÊU THAM KHẢO

1. Đố Như Hơn (2014). Nhãn khoa tập 2, NXB Y hoc, $238-251$.

2. Nguyê̂n Thị Thanh Thu (2002). Nghiên cứu NA trung bình của một nhóm người Việt Nam trưởng thành bằng NAK Goldmann. Luận văn tốt nghiệp thạc sỹ y học, Đại học Y Hà Nội, Hà Nội.

3. Ayres Mdos S (1968). Comparative study between the Goldmann and Maclakov tonometers. Rev Bras Oftalmol, 27(1), 57-68.

4. Võ Đức Dũng, Trân Thị Phương Thu (2005). So sánh kết quả NA đo bằng NAK Goldmann và NAK Maclakov.Y Hoc TP. Hồ Chí Minh, tập 9 phụ bản của Số 1, 55-59.

5. Pham Minh Đức, Đoàn Trong Hâu (2003), So sánh kết quả NA đo bằng NAK khổng tiếp xúc và Maclakov ở người Viêt Nam bình thường trên 20 tuổi, tạp chí Y Học TP. Hồ Chí Minh,tập 7 Phụ bản số 1.

\title{
ĐÁNH GIÁ HIỂU QUẢ PHƯƠNG PHÁP TIÊM NONG KHỚP DƯỚI DSA ĐIỀU TRI BỆNH ĐÔNG CỨNG KHỚP VAI BẰNG THANG ĐIỂM SPADI
}

\author{
Trần Anh Tuấn ${ }^{1}$, Nguyễn Thị Thu Hằng ${ }^{1}$, Nguyễn Duy Trinh ${ }^{1}$
}

\section{TÓM TĂT}

Mục tiêu nghiên cứu: Nghiên cứu hiệu quả điều trị bơm nong khớp vai dưới DSA bằng thang điểm SPADI và các yếu tố ảnh hưởng. Đối tượng và phương pháp: Các bênh nhân đông cứng khớp vai được bơm nong ổ khớp dưới DSA, dựa trên lượng thuốc bơm, mức độ ngấm các buồng khớp để đánh giá mức đố hep và lương thuốc cần thiêt để nong khớp, đánh giá mức độ cải thiện triệu chứng lâm sàng. Kết quả: Có 38 khớp vai được bớm nong, trong đó các buống hẹp chính là buồng dưới $(84,2 \%)$, rãnh nhị đầu $(94,7 \%)$. Mức độ hẹp nặng và trung bình lần lượt 34,2\% và 52,6\%. Các buồng được nong đạt tỷ lệ cao tữ $77,8 \%$ tói $100 \%$ (buông trển). Thang điểm SPADI giảm 20,03 $\pm 7,81$ điểm sau 2 tuân và $32,47 \pm 10,80$ sau 4 tuần. Tuổi trẻ và điều trị sớm là hai yếu tố cho kết quả tiên lượng điều trị tốt hơn. Kết luâan: Bớm nong ổ khớp điều trị bệnh đông cứng khớp vai dưới DSA là an toàn và đạt hiệu quản cao, thang điểm SPADI dễ áp dụng đánh giá hiệu quả điều trị.

Tư khóa: Đông cứng khớp vai, ȘPADI, bơm nong.

\section{SUMMARY}

EVALUATION THE EFFECTIVENESS OF FLUOROSCOPIC-GUIDED HYDRODILATATION IN THE TREATMENT OF FROZEN SHOULDER BASED ON SPADI SCORES

\footnotetext{
${ }^{1}$ Trung tâm điên quang, BV Bach Mai Chịu trách nhiệm chính: Trần Anh Tuấn Email: Bs.trananhtuan@yahoo.com.vn Ngày nhận bài: 21.12.2020 Ngày phản biện khoa học: 25.01.2021 Ngày duyệt bài: 2.2.2021
}

Objective: This study was conduted to evaluate
the effectiveness of fluoroscopic-guided hydrodilatation in the treatment of frozen shoulder based on Shoulder Pain and Disability Index (SPADI) scores and other factors. Material and method: The patients diagnosed with frozen sholder disease were hydrodilatated with corticosteroid injection performed via an anterior approach under fluoroscopy. Based on the amount of drug pumped, the degree of drug infiltration, we could evaluate the stenosis grade and calculate the medication volume exactly needed for each procedure in combination with level of clinical symptom improvement. Patients were characterized at admission and followed up at at two and four weeks post-intervention with Shoulder Pain and Disability Index (SPADI) scores and measurement of motion active and passive range. Result: A total of 38 consecutive patients with frozen shoulder underwent a distension arthrogram mostly in inferior chamber $(84.2 \%)$ and bicipital sulcus (94.7\%). The mild and severe stenosis were conscutively $34.2 \%$ and $52.6 \%$. The good results post-dilatation ranged from $77.8 \%$ to $100 \%$ (superior chamber). The SPADI score was reduced to $20,03 \pm 7,81$ and $32,47 \pm 10,80$, consecutively, after 2 and 4 weeks post-distension. Coclusion: Our study results consolidated that arthrographic capsular distension progressing is an effective treatment for adhesive capsulitis. This procedure was performed through an anterior-lateral approach under fluoroscopic guidance is accurate, reliable and minimally invasive.

Keywords: adhesive capsulitis, arthographic capsular distension, SPADI

\section{I. ĐĂT VẤN ĐỀ}

Tiêm nong khớp vai dưới hướng dẫn của máy 